\title{
Automatic Bubble Detection in Cardiac Video Imaging
}

\author{
Ismail Burak Parlak, Ahmet Ademoglu, Salih Murat Egi, Costantino Balestra, Peter Germonpre, \\ and Alessandro Marroni
}

\begin{abstract}
Bubble recognition is a challenging problem in a broad range from mechanics to medicine. These gas-filled structures whose pattern and morphology alter in their surrounding environment would be counted either manually or with computational recognition procedures. In cardiology, user dependent bubble detection and temporal counting in videos require special trainings and experience due to ultra fast movement, inherent noise and video quality. In this study, we propose an efficient recognition routine to increase the objectivity of emboli detection. Firstly, we started to compare five different methods on two synthetic data sets emulating cardiac chamber environment with increasing speckle noise levels. Secondly, real echocardiographic video records were segmented by variational active contours and Left Atria (LA) were extracted. Finally, three successful methods in simulation were applied to LAs in order to reveal candidate bubbles on video frames. Our detection rate of proposed method was $95.7 \%$ and the others were $86.2 \%$ and $88.3 \%$. We conclude that our approach would be useful in long lasting video processing and would be applied in other disciplines.
\end{abstract}

Index Terms-Image thresholding, active contours, venous emboli, echocardiography.

\section{INTRODUCTION}

In different disciplines, several approaches are developed to detect bubbles and cavitations. These gas-filled structures are generally formed within objects, surfaces, liquids, thin films and inner layers. In solid state mechanics, thermodynamics and metallurgy, bubbles and cavitations are generally locked and have a non-moving nature. On the other hand, their dynamics in fluids are characterized through the viscosity of surrounding environments and physical properties of inner media. Transition between different layers, the effect of non-newtonian fluids would cause considerable variations in

Manuscript received May 23, 2011. Manuscript accepted for publication August 25, 2011.

I.B. Parlak is with the Institute of Biomedical Engineering, Bogazici University and the Department of Computer Engineering, Galatasaray University, Ciragan Cad. No:36 34257 Istanbul, TURKEY e-mail:bparlak@gsu.edu.tr.

A. Ademoglu is with the Institute of Biomedical Engineering, Bogazici University, Istanbul, TURKEY.

S.M. Egi is with the Department of Computer Engineering, Galatasaray University, Istanbul, TURKEY.

C. Balestra is with the Environmental \& Occupational Physiology Lab., Haute Ecole Paul Henri Spaak, Brussels, BELGIUM.

P. Germonpre is with the Centre for Hyperbaric Oxygen Therapy, Military Hospital, Brussels, BELGIUM.

A. Marroni is with Divers Alert Network (DAN) Europe Research Committee, Brussels, BELGIUM. their behaviors. Even if bubble models offer a generalization for quantification and motion estimation in these fields, bubble monitoring and recognition in medicine still conserve unresolved problems.

In medicine, bubbles so-called emboli are created in endothelial tissues and are transported with veins to the heart. Though the bubble visualization in time is a challenge for an untrained clinician, human wise bubble recognition is a spatial problem due to turbulence, endocardial tissues, blood transportation and especially high level inherent noise. For healthy subjects embolus would be filtered in circulation, lung shunts or at most they would trigger migraine. However in risky groups, they would cause severe diseases in a broad range from stroke to blindness. For this purpose clinicians are mostly focused on Left Atrium (LA) and Pulmonary Artery (PA). Bubble examination is performed through video streams. Echocardiologists refer to manually selected region of interests (ROI) and try to discriminate moving objects which are labeled as bubbles. These moving objects might have nonlinear nature as Postema et al. [1] schematized such as translation, fragmentation, clustering, jetting and cracking.

Initial attempts to detect bubbles in circulation were based to Doppler ultrasonography. Embolus which are travelling through superior vena cava are classified manually as candidate bubbles when a sound peak from baseline or mean in frequency domain is observed.

Computational procedures were developed to automatize these methods which would cause variations between clinicians or mislead grading methods of bubbles for diagnosis purposes [2], [3].

Moreover, different computational approaches in other disciplines proposed recognition solutions for spatially non-moving bubbles or in low noise levels which would not mislead iterative algorithms or cost functions. Snakes[4], [5], [6], contour based models [7], principal component analysis[8], gradient based thresholding methods[9], [10] were utilized by different groups. Even if these methods would give accurate detection results in single frames, they would cause big time delays in videos. Furthermore, we note that speckle noise, low resolution and partial view of cardiac chambers are other bottlenecks in echocardiography.

Threshold based methods would be preferred in video frames if computational processing time does not cause delay and false alarms are low in recognition results. Researchers in medicine developed frame based semi-automatic approaches. 
Brubakk et al. [11] proposed intensity and spatial thresholding algorithm in manually selected ROIs. Norton et al. [12] developed a quantification based on temporal change in opacification through cardiac chambers. These methods brought an expansion in this area as first automated analyzes.

In this study, we developed a spatio-temporal method for bubble quantification. Both simulation and real echogenic records were examined through different procedures with additive noise. In our algorithm, pixel series in acquired frames were thresholded dynamically by separating systolic and diastolic time intervals respectively. Moreover, all detected bubble wise structures were gathered into one frame. On the parallel side four different thresholding procedures were applied onto same data. Performance analysis reveals that our approach is satisfactory through false alarms. We hypothesize that our method provides better detection rates and increases the clinician subjective ease-of-use in terms of decision making in recognition.

\section{Methodology}

\section{A. Simulation Videos}

We started to create two different congenital atrial video records. Simulated frames were set as $160 \times 120$ pixels, the average size of segmented LA in real records. Each video stream was $1 \mathrm{sec}$ long and 25 frames/sec (fps). Bubbles on simulation data were placed randomly as it is in real environment. Their contrasts were close to real embolus. In echocardiographic records, bubbles are travelling dynamically and same bubbles generally might be seen in two previous or posterior frames if there is not a massive opacification. This visual procedure is applied by clinicians to lower false alarms. In order to set same echocardiographic environment and bubble behavior, we either placed bubbles in previous and next frames by translating, rotating or removed. This simulation procedure is checked double blinded by two different clinicians. In order to evaluate the performance of recognition algorithms, we generated speckle noise using uniform distribution. This noise with mean $\mu ; 0$ and different variances $\sigma$ was added to simulation frames. In simulation, we also adopted Germonpre et al. [13] criterion for bubble classification in congenital diseases. When bubble numbers in examined area is more or less than 20 , subjects are grouped as Type 1 and Type 2, respectively.

\section{B. Cardiac Videos}

We acquired two contrast Transoesophageal Echocardiogram (cTEE) video records from two male professional divers. The study protocol was approved in advance by Centre for Hyperbaric Oxygen Therapy, Military Hospital, Ethics Committee. Each subject provided written informed consent to join the study.

Embolus detection and visualization protocol described by Germonpre, et al. [13] is utilized for each subject. Both divers underwent cTEE with agitated saline for contrast.
All cTEE video frames were recorded from Ultrasound device (MicroMaxx, SonoSite Inc, WA) in high definition 640x480 pixels, avi format. For all subjects, acquisition was performed three times to ascertain human based grading by two echocardiologists as a double blind study.

\section{Segmentation}

Initially we started to perform our segmentation using active contours implemented via level set introduced by Caselles et al. [14]. In this approach contours are found using a Lagrangian formulation based on the evolution of parametrized curve. We remarked that the partial differential equation so called evolution is relatively slow in terms of computational time on video sequences. Therefore we adopted a modified level set formulation and combined the methods of Chan et al. and Vemuri et al. [15], [16].

An initial level set by fronts $\Gamma$ is denoted by a distance function $\phi(x)= \pm d_{\Gamma}(x)$ A zero level set function is; $\Gamma(t)=(x, y): \phi(x)=0$ Given the frant $\Gamma$ let $F(x)$ be the speed function in the direction of the normal of $\Gamma$ and $x(t)$ be a point on $\Gamma$ which evolves progressively then $\phi(x(t), t) \equiv 0$ for all $t$. When this expression is differentiated through $t$;

$$
\frac{\partial \phi}{\partial t}+\nabla \phi \frac{d x}{d t}=0
$$

Level set function has both positive and negative terms including zeroes and is called signed distance function;

$$
\frac{\partial \phi}{\partial t}=\operatorname{sign}(\phi)(1-|\nabla \phi|)
$$

We resolved this equation by interpreting without reinitialization. This approach is based on modified formulation that consists of two energy terms; internal and external. Internal term prevents the deviation of level set from signed distance function whereas external term conducts a motion on zero level set up to the final pattern features especially contours. A consequent evolution of this level set is a gradient flow and it minimizes the energy function as it is expressed in Equation 1 and 2. All digital records were segmented and analyzed in MATLAB 2010a (The MathWorks Inc, Massachusetts).

\section{Detection Algorithms}

In the review paper of Sezgin et al.[17], distinctive thresholding algorithms were classified through image analysis within different categories.

For our study, we have used four different thresholding methods; Otsu [18], Yen et al. [19], Ramesh et al. [20] and Beghdadi et al. [21] with distinctive nature on image analysis and recognition. Thresholding algorithms create binary level images using either RGB color or gray level images. After thresholding process, blobs would be recognized on frames.

Cardiac patterns and especially LA are composed of gray level patterns. In our simulation and real echogenic videos, bubbles would be easily identified through relevant 
thresholds. However, it should be remarked that ultrasonic image processing is vulnerable to inherent speckle noise. This type of interference would cause misleading in detection, affect binary level image or introduce false alarms within target blobs. In our study, these blobs correspond to candidate bubbles.

All recognition results from each method were compared with human wise detection in order to perform the statistical rate of recognition. In simulation phase, we remarked that two methods are reliable in bubble detection for different noise levels. Therefore we applied them to real segmented cardiac videos.

\section{E. Proposed Method}

2D Cardiac images form a three dimensional data when frames are acquired sequentially with a device dependent fps. Therefore, all pixels in LA have a time series. It would be foreseen that when a bubble wise structure will be present on this pixel, its gray level will change suddenly. For this reason, a dynamic threshold which is applied to each pixel series would reveal candidate bubbles as it is shown in Fig.3.

This dynamic threshold is set using mean $\mu$ and $\sigma$ of pixel time series. When a pixel value is above $\mu+2 \sigma$, this pixel is recognized as a bubble candidate in time. After this recognition procedure, all bubble candidates are summed up on corresponding frame. After detection, we added all bubbles into one single frame as a novelty. This single frame gathers all candidates and facilitates the visual recognition phase for a clinician. After automatic recognition statistical analysis was performed to compare real bubbles marked by two clinicians with computational recognition.

\section{RESULTS}

In this paper, results are interpreted as simulation and real echogenic bubbles. In both steps, our proposed method offered better accuracy and low false alarms than existent methods.

In the simulation phase five methods including our method were tested on two different congenital forms through increasing noise levels. Simulation results were compared in Table I and Fig. 1. Only three methods were satisfactory in high level noises. Therefore, we selected them to test their performance in real data.

In real echogenic forms, recognition algorithms offered reliable methods as it is shown in Table II. However, existent methods were vulnerable to inherent noise and endocardial structures. It is noted in Fig. 2 that boundary structures could not be thresholded efficiently with Ramesh et al.[20] or Yen et al.[19]. Our proposed method detects bubbles with low false alarms. It is also evident that bubble detection map which bring all recognized candidates into one frame is a novelty in this paper. In Fig. 4, the visualization of all detected microembolus onto one single frame brings an ease-of-use for bubble movements.

\section{DisCUSSION \& CONCLUSION}

Bubbles would be recognized accurately with different approaches in steady state environments without noise. On the other hand, medical imaging introduces always artifacts and inherent noise. Microemboli in cardiology are affected with speckle noise and their patterns are close to boundary structures. Therefore, their recognition which is a diagnostic tool for specialists in cardiology becomes a challenging problem in video processing.

In real echogenic frames, blood circulation in LA translates and rotates bubbles. They would be recognized as easily as in simulated frames in Fig. 1. However, during the circulation bubbles would be clustered, cracked or fragmented due their physical properties and turbulence. In these cases, a severe blurring causes false alarms. Moreover, it is noted that some fragments of endocardial wall would be detected as bubbles. In Fig. 2, recognition with Yen et al.[19] and Ramesh et al.[20] could not filter out endocardial fragments. Suboptimal image quality and acoustic shadowing which are the main artifacts in echogenic records are also another challenge for detection. They lower detection rates by inserting dashed or circular spots whose contrast is identical with real bubbles.

Our method benefits a reliable detection as it is noted in Table I and II with low false alarms. The only pitfall of proposed method is its computational time. It is evident that all pixel series should be interpreted to create frames containing only blobs; candidate bubbles.

As a feature work, we note that bubble behavior would be studied in other cardiac chambers or pulmonary artery using other imaging modalities to build up a computational framework in cardiac analysis.

\section{ACKNOWLEDGMENT}

The authors would like to thank Galatasaray and Bogazici Universities for their academical supports and also Divers Alert Network (DAN) for echocardiographic setup and imaging modalities.

\section{REFERENCES}

[1] M. Postema and O. H. Gilja, "Contrast-enhanced and targeted ultrasound," World J Gastroenterol, vol. 17, 2011, pp. 28-41.

[2] K. Tufan, A. Ademoglu, E. Kurtaran, G. Yildiz, S. Aydin and S. M. Egi, "Automatic detection of bubbles in the subclavian vein using doppler ultrasound signals," Aviat Space Environ Med., vol. 77, 2006, pp. 957-962.

[3] H. Nakamura, Y. Inoue, T. Kudo, N. Kurihara, N. Sugano and T. Iwai, "Detection of venous emboli using doppler ultrasound," European Journal of Vascular \& Endovascular Surgery, vol. 35, 2008, pp. 96-101.

[4] J. Wang, X. Huang and Y. Zou, "Bubble detection of railway castings based on snake model," Jisuanji Gongcheng / Computer Engineering, vol. 36, 2010, pp. 205-207.

[5] K. H. Chung, M. J Simmons and M. Barigou, "Local gas and liquid phase velocity measurement in a miniature stirred vessel using PIV combined with a new image processing algorithm," Experimental Thermal and Fluid Science, vol. 33, 2009, pp. 743-753.

[6] D. C. Cheng and H. Burkhardt, "Bubble recognition from image sequences," in Inverse Problems and Experimental Design in Thermal and Mechanical Engineering, Eurotherm Seminar N. 68, 2001.

[7] D. C. Cheng and H. Burkhardt, "Bubble tracking in image sequences," International Journal of Thermal Sciences, vol. 42, 2003, pp. 647-655. 
TABLE I

EVALUATION OF DETECTION RESULTS IN SIMULATED DATA

\begin{tabular}{|c|c|c|c|c|c|c|}
\hline & \multicolumn{3}{|c|}{ Simulation1 } & \multicolumn{3}{c|}{ Simulation2 } \\
& Original & $\sigma=0.07$ & $\sigma=0.5$ & Original & $\sigma=0.07$ & $\sigma=0.5$ \\
\hline Otsu[18] & $100 \%$ & $96.7 \%$ & $6.4 \%$ & $100 \%$ & $97.3 \%$ & $3.2 \%$ \\
Yen et al.[19] & $100 \%$ & $99.1 \%$ & $97.5 \%$ & $100 \%$ & $98.4 \%$ & $97.2 \%$ \\
Ramesh et al.[20] & $98.7 \%$ & $98.3 \%$ & $96.7 \%$ & $100 \%$ & $99.1 \%$ & $97.4 \%$ \\
Beghdadi et al.[21] & $99.6 \%$ & $5.2 \%$ & $0 \%$ & $100 \%$ & $9.5 \%$ & $0 \%$ \\
Proposed method & $100 \%$ & $98.8 \%$ & $97.2 \%$ & $100 \%$ & $99.3 \%$ & $98.1 \%$ \\
\hline
\end{tabular}

TABLE II

EVALUATION OF DETECTION RESULTS IN REAL CARDIAC VIDEOS

\begin{tabular}{|c|c|c|c|}
\hline & $\begin{array}{c}\text { Video 1 } \\
\text { Detection Ration }\end{array}$ & $\begin{array}{c}\text { Video 2 } \\
\text { Detection Ration }\end{array}$ & Mean Ratio \\
\hline Ramesh et al.[20] & $85.8 \%$ & $87.1 \%$ & $86.2 \%$ \\
Yen et al.[19] & $87.6 \%$ & $88.9 \%$ & $88.3 \%$ \\
Proposed method & $93.8 \%$ & $96.5 \%$ & $95.7 \%$ \\
\hline
\end{tabular}

[8] D. C. Cheng and H. Burkhardt, "Template-based bubble identification and tracking in image sequences," International Journal of Thermal Sciences, vol. 45,2006 , pp. 321-330.

[9] M. Honkanen, H. Eloranta and P. Saarenrinne, "Digital imaging measurement of dense multiphase flows in industrial processes," Flow Measurement and Instrumentation, vol. 21, 2010, pp. 25-32.

[10] M. Honkanen, P. Saarenrinne, T. Stoor and J. Niinimaki, "Recognition of highly overlapping ellipse-like bubble images," Measurement Science and Technology, vol. 16, 2005, pp. 1760-1770.

[11] O. Eftedal and A. O. Brubakk, "Detecting intravascular gas bubbles in ultrasonic images," Med Biol Eng Comput. vol. 31, 1993, pp. 627-633.

[12] M. S. Norton, A. J. Sims, D. Morris, T. Zaglavara, M. A. Kenny and A. Murray, "Quantification of echo contrast passage across a patent foramen ovale," in:Computers in Cardiology, IEEE Press, 1998, pp. $89-92$.

[13] P. Germonpre, P. Dendale, P. Unger and C. Balestra, "Patent foramen ovale and decompression sickness in sports divers," Journal of Applied Physiology, vol. 84, 1998, pp. 1622-1626.

[14] V. Caselles, F. Catte, T. Coll and F. Dibos, "A geometric model for active contours and image processing," Numer. Math. vol. 66, 1993, pp. 1-31.

[15] T. Chan and L. Vese, "Active contours without edges," IEEE Trans Image Process, vol. 10, 2001, pp. 266-277.

[16] B. Vemuri and Y. Chen, "Joint image registration and segmentation," in:Geometric Level Set Methods in Imaging, Vision and Graphics, Springer, 2003, pp. 251-259.

[17] M. Sezgin and B. Sankur, "Survey over image thresholding techniques and quantitative performance evaluation," in:Journal of Electronic Imaging, vol. 13, 2004, pp. 146-165.

[18] N. Otsu, "A thresholding selection method from gray-level histogram," IEEE Transactions on Systems, Man and Cybernetics, vol. 9, 1979, pp. $62-66$.

[19] J. C. Yen, F. J. Chang and S. Chang, "A new criterion for automatic multilevel thresholding," IEEE Trans. Image Process. vol. 4, 1995, pp. 370-378.

[20] N. Ramesh, J. H. Yoo and I. K. Sethi, "Thresholding based on histogram approximation," IEEE Proceedings Vision, Image and Signal Process. vol. 142, 1995, pp. 271-279.

[21] A. Beghdadi, A. L. Negrate and P. V. De Lesegno, "Entropic thresholding using a block source model," Graphical Models in Image Processing vol. 57, 1995, pp. 197-205. 


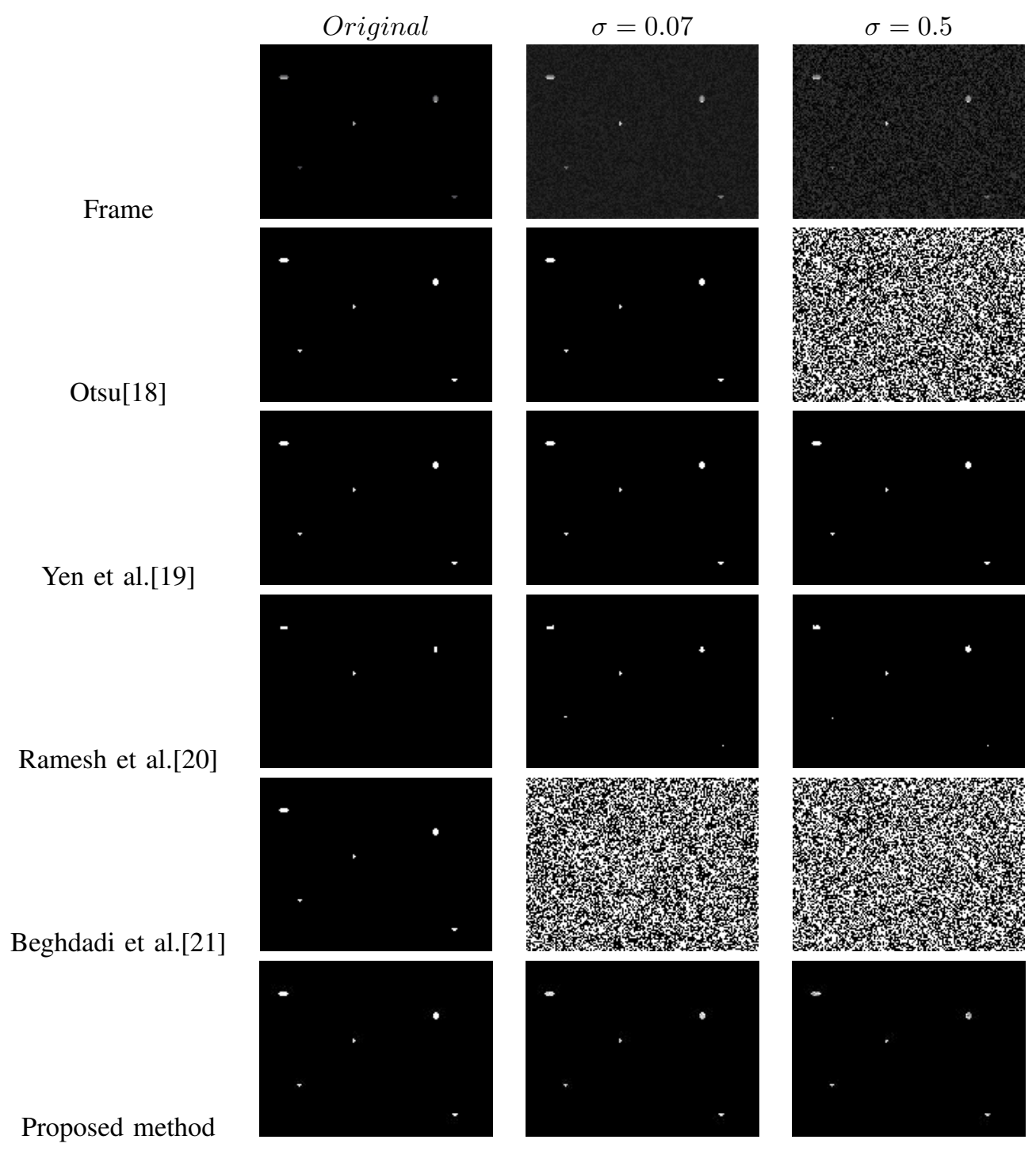

Fig. 1. Comparison of five different methods in simulated LA. 


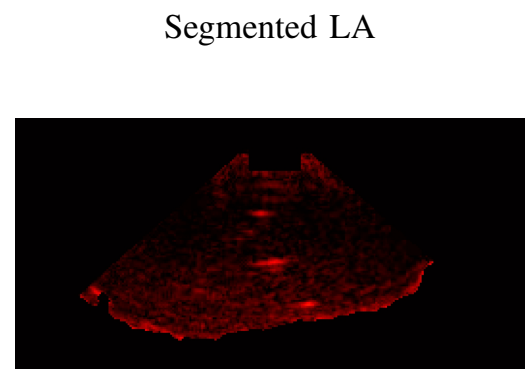

Yen et al.[19]

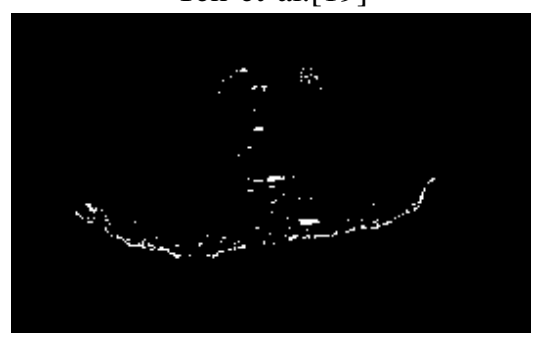

Fig. 2. Comparison of three different methods in TEE.

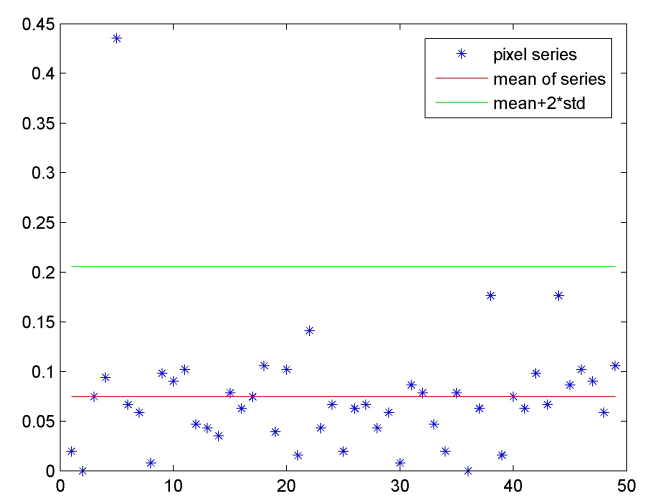

Fig. 3. Dynamic thresholding of pixel through intensity in proposed method.

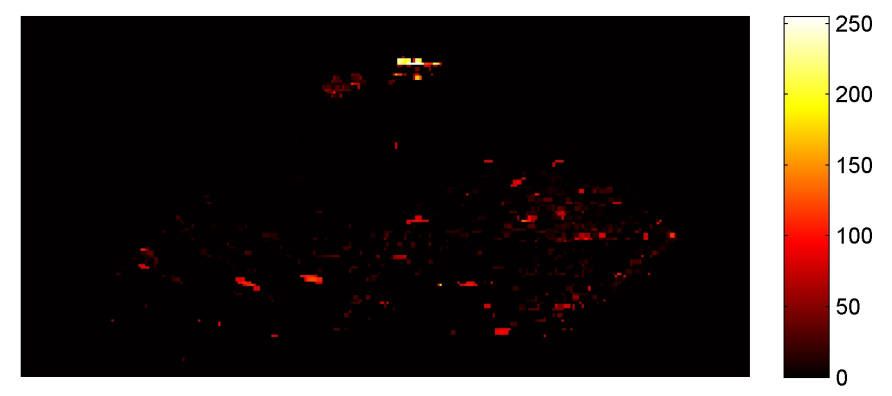

Fig. 4. Bubble map of video sequence. 